\title{
Article
}

\section{Survival Signature-based Reliability Approach for Complex Systems Susceptible to Common Cause Failures}

Feng, Geng

Available at http://clok.uclan.ac.uk/25934/

Feng, Geng ORCID: 0000-0002-4705-3252 (2019) Survival Signature-based Reliability Approach for Complex Systems Susceptible to Common Cause Failures. International Journal of Reliability, Quality and Safety Engineering, 26 (3). p. 1950013. ISSN 0218-5393

It is advisable to refer to the publisher's version if you intend to cite from the work. http://dx.doi.org/10.1142/5021853931950013x

For more information about UCLan's research in this area go to http://www.uclan.ac.uk/researchgroups/ and search for <name of research Group>.

For information about Research generally at UCLan please go to http://www.uclan.ac.uk/research/

All outputs in CLoK are protected by Intellectual Property Rights law, including Copyright law. Copyright, IPR and Moral Rights for the works on this site are retained by the individual authors and/or other copyright owners. Terms and conditions for use of this material are defined in the policies page.

\section{CLoK}

Central Lancashire online Knowledge www.clok.uclan.ac.uk

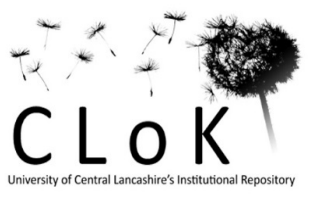




\title{
Article
}

\section{Survival Signature-based Reliability Approach for Complex Systems Susceptible to Common Cause Failures}

\author{
Feng, Geng \\ Available at http://clok.uclan.ac.uk/26139/ \\ Feng, Geng (2019) Survival Signature-based Reliability Approach for Complex Systems \\ Susceptible to Common Cause Failures. International Journal of Reliability, Quality and Safety \\ Engineering . ISSN 0218-5393
}

It is advisable to refer to the publisher's version if you intend to cite from the work. http://dx.doi.org/10.1142/S021853931950013X

For more information about UCLan's research in this area go to http://www.uclan.ac.uk/researchgroups/ and search for < name of research Group>.

For information about Research generally at UCLan please go to http://www.uclan.ac.uk/research/

All outputs in CLoK are protected by Intellectual Property Rights law, including Copyright law. Copyright, IPR and Moral Rights for the works on this site are retained by the individual authors and/or other copyright owners. Terms and conditions for use of this material are defined in the http://clok.uclan.ac.uk/policies/

\section{CLoK}

Central Lancashire online Knowledge www.clok.uclan.ac.uk

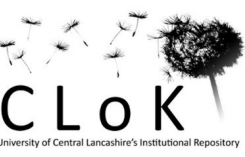


International Journal of Reliability, Quality and Safety Engineering

(c) World Scientific Publishing Company

\title{
Survival Signature Based Reliability Approach for Complex Systems Susceptible to Common Cause Failures
}

\author{
Geng Feng \\ School of Engineering \\ University of Central Lancashire \\ Preston, PR1 2HE, United Kingdom \\ gfeng@uclan.ac.uk \\ Received (Day Month Year) \\ Revised (Day Month Year)
}

\begin{abstract}
The importance of reliability to complex systems cannot be disputed as they are the backbones of our society. In practice, the common cause failures may have severe reverse function on complex systems' overall stability. Survival Signature opens a new way to perform reliability analysis on systems with multiple component types. This paper takes a research on survival signature based reliability analysis on complex systems susceptible to Common Cause Failures. To be specific, it proposes the standard $\alpha$-factor model and general $\alpha$-factor model to combine with the survival signature. In practical applications, the $\alpha$-factor estimator of the system might not be defined completely due to limited data, or knowledge which requires to take imprecision into account. Some numerical cases are presented to show the applicability of the methods for complex systems. In addition, this paper may attract people's attention on the conception of Design for Reliability.

Keywords: Common Cause Failures; Survival Signature; $\alpha$-Factor Model; Reliability Analysis; Complex Systems.
\end{abstract}

\section{Introduction}

A common cause failure $(\mathrm{CCF})$ is an event that causes multiple components fail simultaneously, which exists widely in complex systems. Modelling CCFs is essential in complex systems reliability analysis as they can have a large effect on the systems' overall functionality, especially on the quality and productivity of products and processes. ${ }^{1}$ There is always an assumption that the components failures are independent, however, CCFs make the hypothesis is not set up. What is more, CCFs have been shown by many studies to decrease the reliability and availability of complex systems. Therefore, common cause failures are extremely important in reliability assessment and must be given adequate treatment to minimize gross overestimation of performances. ${ }^{2}$

A number of parametric models have been developed for common cause failures over the last decades. Rasmuson and Kelly reviewed the basic concepts of modelling CCFs in reliability and risk studies. ${ }^{3}$ One of the most commonly used 
single parameter models defined by Fleming ${ }^{4}$ is called the $\beta$-factor model, which is the first parameter model applied to common cause failures in risk and reliability analysis. Then he generalized the $\beta$-factor model to multiple Greek letter model in $1986 .{ }^{5}$ The $\alpha$-factor model which is proposed by Mosleh et al. ${ }^{6,7}$ develops CCFs from a set of failure ratios and the total component failure rate. The binomial failure rate model discussed by Atwood ${ }^{8}$ estimates the failure frequency of two or more components in a redundant system as the product of the CCF shock arrival rate and the conditional failure probability of components given the shock has occurred. A system's components each contribute differently to the system, which are defined as weights. ${ }^{9}$ Systems with weighted components are useful to model various capacity-based engineering systems such as oil transportation system, power generation system, and production system. ${ }^{10}$ Different from weighted reliability components measure, which mainly focus on the influence of different weighted components on the reliability of system, $\alpha$-factor model is mainly used for estimating the probability of how many components fail due to a common cause failure event.

Recently, based on the $\alpha$-factor model, Kelly and Atwood ${ }^{11}$ presented a method for developing Dirichlet prior distributions that have specified marginal means, but which are otherwise minimally informative. An explicit method and an implicit method is proposed $\mathrm{in}^{12}$ to analyse the reliability of systems subject to internal or external probabilistic common cause failures. A general applicable and effiecient Monte Carlo simulation approach is used for reliability evaluation of systems susceptible to induced failures in. ${ }^{13}$ Levitin ${ }^{14}$ incorporated the common cause faliures into non-repairable multistate series-parallel system analysis by using the universal generating function method. A robust Bayesian approach to modelling epistemic uncertainty in the imprecise Dirichlet model has been discussed by Troffaes et al. ${ }^{15}$ Coolen and Coolen-Maturi ${ }^{16}$ presents non-parametric predictive inference for system reliability following common cause failures of components. In that paper, attention is restricted to system with exchangeable or a single type of components. Therefore, an extension of this work is needed. To be specific, it is necessary to perform reliability analysis on complex systems by considering CCFs among components belonging to different types. Survival signature provides a good way solve this problem.

Survival signature was first proposed by Coolen and Coolen-Maturi ${ }^{17}$ in 2012. It is a powerful methodology that can not only hold the merits of the former system signature, ${ }^{18}$ but can be used in complex system with components belong to multiple types. In essence, it does not have the assumption that components of different types are exchangeable, which overcomes the long-standing limitation of the system signature. This is useful when a system which may have components with failure times that follow different probability distributions. ${ }^{19,20}$ Therefore, survival signature is a promising method for application to complex systems and networks.

Based on the former work, Aslett developed a Reliability Theory package which 
was used to calculate the survival signature ${ }^{21}$ and analysed system reliability within the Bayesian framework of statistics. ${ }^{22}$ A non-parametric predictive inference for system reliability using the survival signature was proposed by Coolen et al. ${ }^{23}$ Feng et al. deals with the imprecision within the system by analytical and numerical ways respectively, ${ }^{24}$ what is more, probability bounds analysis is used to perform sensitivity analysis on complex systems with epistemi uncertainty in the paper. ${ }^{25}$ An imprecise Bayesian non-parametric approach by using sets of priors to system reliability with multiple types of components is developed by Walter et al. ${ }^{26}$ Patelli et al. ${ }^{27}$ proposed efficient simulation approaches which are based on survival signature for reliability analysis on large system. An efficient algorithm for the exact computation of the survival signature of large systems was put forward by Reed. ${ }^{28}$ Eryilmaz et al. ${ }^{29}$ developed the survival signature-based marginal and joint reliability importance measures to optimise system design, as well as consider mean residual life of coherent systems with multiple types of dependent components in the article. ${ }^{30}$

A survival signature based reliability analysis on complex systems with common cause failures has been proposed in this paper. The $\alpha$-factor model distinct between the total failure rate of a component and the common cause failures modelled by $\alpha$-factor parameters, which can be got through experts' judgement of the system or the past data on the system. These advantages of the $\alpha$-factor model make it possible to combine with the survival signature to assess the complex system reliability. The standard $\alpha$-factor model is proposed first in the paper to perform system reliability analysis after CCFs, however, it has two assumptions that there must be at least one component fails of each type and the components failures of each type are independent. In order to remove these assumptions, another general $\alpha$-factor model has been introduced. It takes all the possible combinations of the failed numbers of past events. The novel standard and general $\alpha$-factor models can be expressed by the equations which connect with the survival signature. What is more, the imprecision within the models are also considered. The applicability of the proposed approaches are demonstrated by solving the numerical cases, by investigating the results, the concept of design for reliability should be drawn more attention.

This paper is organized as follows. Section 2 gives a brief conceptions about the $\alpha$-factor model and survival signature. The system reliability analysis after common cause failures has been presented in Section 3. In this Section, the standard $\alpha$ factor model and the general $\alpha$-factor model have been studied respectively. The applicability and performance of the proposed approaches is presented in Section 4. Finally Section 5 closes the paper with conclusions and discussion. 


\section{2. $\alpha$-Factor Model and Survival Signature}

\section{1. $\alpha$-factor model}

The $\alpha$-factor model is particularly useful in the practical engineering world as the alpha factor parameters can be got through experts' judgement of the system or past data on the system. The parameters $\alpha_{k}$ of the model are the fractions of the total probability of failure in the system that involves the failure of $k$ components due to a common cause.

The probability of a common cause basic event involving failure of $k$ components in a system of $m$ components $^{31}$ can be calculated by Equation 1 .

$$
Q_{k}=\frac{k}{\left(\begin{array}{c}
m-1 \\
k-1
\end{array}\right)} \frac{\alpha_{k}}{\alpha_{t}} Q_{t}
$$

where, $k=1,2, \ldots, m$ and $\alpha_{t}=\sum_{k=1}^{m} k \alpha_{k} . Q_{t}$ is the total probability of failure accounting both for common cause failures and independent failures. The alpha parameter estimator can be expressed as:

$$
\alpha_{k}=\frac{n_{k}}{\sum_{i=1}^{m} n_{i}}
$$

where, $n_{k}$ is the number of events with $k$ failed components.

The alpha parameter estimator represents the probability that exactly $k$ of the $m$ components fail, given that at least one failure occurs. It can be seen from Equation 2 that the sum of the $\alpha_{k}$ will be 1 . The advantage of the $\alpha$-factor model is its distinction between the total failure rate of a component $Q_{t}$, for which we generally have a lot of information, and common cause failures modelled by $\alpha_{k}$, for which we generally have very little information. ${ }^{15}$

\subsection{Survival signature}

Suppose there is a system with $m$ components which belong to $K \geq 2$ component types, with $m_{k}$ components of type $k \in\{1,2, \ldots, K\}$ and $\sum_{k=1}^{K} m_{k}=m$. Assume that the random failure times of components of the system type are exchangeable, while full independence is assumed for components belong to different types $(i i d)$, Coolen ${ }^{17}$ proposed the survival signature which can be denoted by $\Phi\left(l_{1}, l_{2}, \ldots, l_{K}\right)$, with $l_{k}=0,1, \ldots, m_{k}$ for $k=1,2, \ldots, K$. It defines the probability that the system functions given that $l_{k}$ of its $m_{k}$ components of type $k$ work, for each $k \in\{1,2, \ldots, K\}$. There are $\left(\begin{array}{c}m_{k} \\ l_{k}\end{array}\right)$ state vectors $\underline{x}^{k}$ with $\sum_{i=1}^{m_{k}} x_{i}^{k}=l_{k}$ $(k=1,2, \ldots, K)$, where $\underline{x}^{k}=\left(x_{1}^{k}, x_{2}^{k}, \ldots, x_{m_{k}}^{k}\right)$. Let $S_{l_{1}, l_{2}, \ldots, l_{K}}$ denote the set of all state vectors for the whole system, and it can be known that all the state vectors $\underline{x}^{k} \in S_{l_{k}}^{k}$ are equally likely to occur. Therefore, the survival signature can be expressed as: 


$$
\Phi\left(l_{1}, \ldots, l_{K}\right)=\left[\prod_{k=1}^{K}\left(\begin{array}{c}
m_{k} \\
l_{k}
\end{array}\right)^{-1}\right] \times \sum_{\underline{x} \in S_{l_{1}, \ldots, l_{K}}} \phi(\underline{x})
$$

Let $C_{k}(t) \in\left\{0,1, \ldots, m_{k}\right\}$ denote the number of $k$ components working at time $t$. Assume that the components of type $k$ have a known cumulative distribution function $(C D F) F_{k}(t)$ and the components failure times of different type are assumed independent, then:

$$
P\left(\bigcap_{k=1}^{K}\left\{C_{k}(t)=l_{k}\right\}\right)=\prod_{k=1}^{K} P\left(C_{k}(t)=l_{k}\right)=\prod_{k=1}^{K}\left(\begin{array}{c}
m_{k} \\
l_{k}
\end{array}\right)\left[F_{k}(t)\right]^{m_{k}-l_{k}}\left[1-F_{k}(t)\right]^{l_{k}}(4)
$$

Hence, the survival function of the system with $K$ types of components becomes:

$$
P\left(T_{s}>t\right)=\sum_{l_{1}=0}^{m_{1}} \ldots \sum_{l_{K}=0}^{m_{K}} \Phi\left(l_{1}, \ldots, l_{K}\right) P\left(\bigcap_{k=1}^{K}\left\{C_{k}(t)=l_{k}\right\}\right)
$$

Equation 5 shows that the structure of the system is separated from the its components failure times, which is the typical advantage of the survival signature. The survival signature is a summary of structure functions and only needs to be calculated once for the same system. As a result, it is an efficient method to perform system reliability analysis on complex systems with multiple component types.

\section{System Reliability after Common Cause Failures}

In this section, the survival signature is introduced to analyse complex system with common cause failures. Based on the results of, ${ }^{16}$ the $\alpha$-factor model can be applied to calculate system reliability in the presence of common cause failures.

\subsection{Standard $\alpha$-factor model}

Let assume that there is a system with $m_{k}$ components belong to type $k \in$ $\{1,2, \ldots, K\}$. When a failure event occur, $P\left(f_{1}, f_{2}, \ldots, f_{K}\right)$ denotes the probability that how many failures occur of each component type. The survival signature $\Phi\left(l_{1}, l_{2}, \ldots, l_{K}\right)$ is the probability that the system functions if $l_{k}$ components of type $k$ are working, which can be expressed as $\Phi\left(l_{1}, l_{2}, \ldots, l_{K}\right)=P($ system functions $\mid$ $l_{k}$ components of type $k$ work), where $k \in\{1,2, \ldots, K\}$.

Let $P\left(T_{s}>t \mid C C F\right)$ express the probability that the system still functions after the next common cause failure event, therefore, it can be calculated by

$$
P\left(T_{s}>t \mid C C F\right)=\sum_{l_{1}=0}^{m_{1}} \ldots \sum_{l_{K}=0}^{m_{K}} \Phi\left(l_{1}, \ldots, l_{K}\right) P\left(m_{1}-l_{1}, \ldots, m_{K}-l_{K}\right)
$$


where $f_{i}=m_{i}-l_{i}$.

It can be seen from Equation 6 that unlike the $P\left(T_{s}>t\right)$ calculated by Equation 5, the survival function of the system after the CCFs is independent of $t$. What is more, the typical merit of survival signature can also hold. To be specific, the survival signature, which encompasses information of the system structures, and the probability distribution, which relies on the common cause failures of the components belong to different types, is separated in the equation.

The $\alpha$-factor model with estimate of the alpha factor parameters are mainly used in this section. And they are given by Equation 2 for a common cause group of $m$ components.

$P\left(f_{1}, f_{2}, \ldots, f_{K}\right)$ is constructed by using the past data available on the system, combined with the $\alpha$-factor model for CCFs. For this standard $\alpha$-factor model, there are assumptions that $P(0,0, \ldots, 0)=P\left(0, f_{2}, \ldots, f_{K}\right)=P\left(f_{1}, 0, \ldots, f_{K}\right)=\ldots=$ $P\left(f_{1}, f_{2}, \ldots, 0\right)=0$, as there must be at least one component fails of each type. What is more, there is another assumption that the components failures of each type are independent, which implies that $P\left(f_{1}, f_{2}, \ldots, f_{K}\right)=P\left(f_{1}\right) * P\left(f_{2}\right) * \ldots * P\left(f_{K}\right)$.

Recall that $\alpha_{n_{k}}^{k}$ gives the probability that exactly $n_{k}$ components fail, given that they belong to type $k$. For example, it is assumed that there is 1 component of type 1 failure, it follows that $P\left(f_{1}=1\right)=\alpha_{1}^{1}$. Thus, the alpha parameters provide all of the information required to specify the distribution. For instance, $P(1,1, \ldots, 1)=\alpha_{1}^{1} \alpha_{1}^{2} \ldots \alpha_{1}^{K}$.

The next step is to calculate the survival signature, which will then combine with the $P\left(f_{1}, f_{2}, \ldots, f_{K}\right)$ to assess the survival function of the system. It is not necessary to computer all the survival signatures as it can identify which values are required by Equation 6 .

\subsection{General $\alpha$-factor model}

The standard $\alpha$-factor model may lead to an unsatisfactory low probability level of the system reliability, which due to the assumption that the first failure event will affect components of all types. Therefore, it is essential to find a general $\alpha$-factor model that can avoid the assumption that the failure event has necessarily affected all common cause groups of components.

Let assume there are $K$ common cause component groups, with group $k \in$ $\{1,2, \ldots, K\}$ includes $m_{k}$ components. The past data $n_{j_{1}, j_{2}, \ldots, j_{K}}$ denotes the number of past events with exactly $j_{1}$ failed components from group 1 , exactly $j_{2}$ failure components from group 2 and the like.

The $\alpha$-factor parameter $\alpha_{j_{1}, j_{2}, \ldots, j_{K}}$ provides the probability that exactly $j_{k}$ components of group $k$ fail due to a common cause failure event, with $k \in\{1,2, \ldots, K\}$. Given that a common cause failure event has affected the overall system, however, which exact common cause groups are affected are not known. Therefore, the $\alpha_{j_{1}, j_{2}, \ldots, j_{K}}$ can be estimated by Equation 7. 


$$
\alpha_{j_{1}, \ldots, j_{K}}=\frac{n_{j_{1}, \ldots, j_{K}}}{\left(\sum_{j_{1}=0}^{m_{1}} \cdots \sum_{j_{K}=0}^{m_{K}} n_{j_{1}, \ldots, j_{K}}\right)-n_{0, \ldots, 0}}
$$

The $\sum_{j_{1}=0}^{m_{1}} \ldots \sum_{j_{K}=0}^{m_{K}} n_{j_{1}, \ldots, j_{K}}$ in denominator represents all the possible outcomes, but $n_{0, \ldots, 0}$ has to be subtracted as there has an assumption that at least one component of type $k$ in the system is affected by the common cause failure event. However, this assumption is only valid if $n_{0, \ldots, 0}$ is included in the summation. In practice, there may be no data for the number of no components have failed, therefore, for this circumstance, $n_{0, \ldots, 0}$ is arbitrarily setted as 0 .

The probability of the system works after a common cause failure event can also be expressed by Equation 6 . The survival signature $\Phi\left(l_{1}, \ldots, l_{K}\right)$ will not change for the same system, as it only depends on the structures of the system. However, the joint probability distribution $P\left(f_{1}, f_{2}, \ldots, f_{K}\right)$ is obtained without assuming independence among components, as it is simply given by the alpha factor parameters. To be specific, that means $P\left(f_{1}, f_{2}, \ldots, f_{K}\right)=\alpha_{j_{1}, j_{2}, \ldots, j_{K}}$.

\subsection{Imprecise system reliability after common cause failures}

In the application engineering world, if the system has not been in operation in the past (e.g., is a new system), or it does not usually encounter common cause failure events. All of which means there may not be enough data or perfect inspections to estimate the values of the accurate $\alpha$-factor parameters. ${ }^{32}$ In addition, experts can provide a estimation of the number $n_{k}$ of common cause components failures, but often only an interval is predicted.

Therefore, the $\alpha$-factor parameters might have imprecise values with $\left[\underline{\alpha}_{m_{k}}^{k}, \bar{\alpha}_{m_{k}}^{k}\right]$. Since $P\left(f_{1}, f_{2}, \ldots, f_{K}\right)$ is estimated based on the $\alpha$-factor model, the imprecision will propagate to $P\left(m_{1}-l_{1}, \ldots, m_{K}-l_{K}\right)$. The survival signature remains unaffected by the imprecision since it is only influenced by uncertainty and imprecision in the system structure. Hence, the bounds of the survival probability are calculated as

$$
\begin{aligned}
& \underline{P}\left(S_{C C F}\right)=\sum_{l_{1}=0}^{m_{1}} \ldots \sum_{l_{K}=0}^{m_{K}} \Phi\left(l_{1}, \ldots, l_{K}\right) \underline{P}\left(m_{1}-l_{1}, \ldots, m_{K}-l_{K}\right) \\
& \bar{P}\left(S_{C C F}\right)=\sum_{l_{1}=0}^{m_{1}} \ldots \sum_{l_{K}=0}^{m_{K}} \Phi\left(l_{1}, \ldots, l_{K}\right) \bar{P}\left(m_{1}-l_{1}, \ldots, m_{K}-l_{K}\right)
\end{aligned}
$$

\section{Numerical Examples}

Figure 1 shows a complex system with thirteen components which belong to four types. 


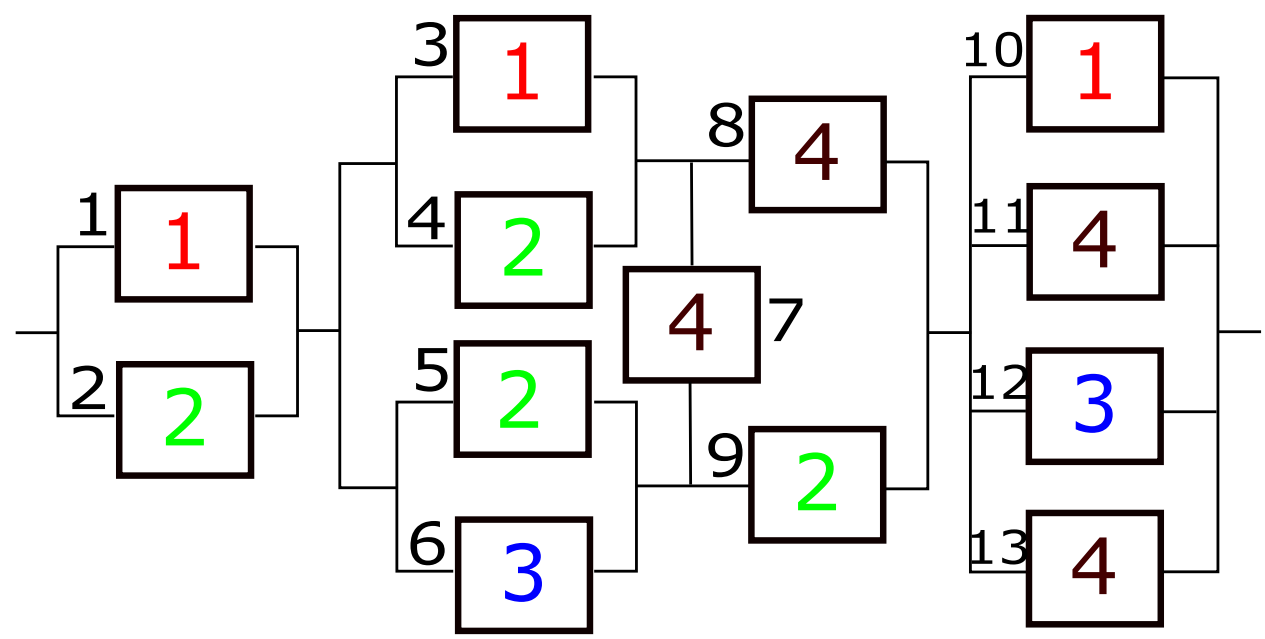

Fig. 1. Complex system with thirteen components which belong to four types. The number inside the component box represents the type, while the number outside the box expresses the component index.

\subsection{Case 1: standard $\alpha$-factor model}

Firstly, let determine the values of the survival function by using the standard $\alpha$-factor model with past data on the complex system. Suppose that the common cause failure groups are the same as component types, this is logical in engineering world as the components of the same type have similar characteristics, therefore, they are more likely to be influenced by the same common cause event.

There have $n_{1}=1, n_{2}=2, n_{3}=1$ for component type 1 . For example, $n_{1}=1$ means that there has been 1 previous occurrences of failure with just one component of type 1 . So the $\alpha$-factor parameter estimator in this case is

$$
\alpha_{1}^{1}=\frac{n_{1}}{n_{1}+n_{2}+n_{3}}=\frac{1}{4}
$$

It can also get the results that $\alpha_{2}^{1}=\frac{1}{2}$ and $\alpha_{3}^{1}=\frac{1}{4}$ in the same way. Just as the said before in this paper, it can be seen the summation of the $\alpha$-factor parameters is equal to 1 .

For type $2, n_{1}=2, n_{2}=1, n_{3}=1$ and $n_{4}=2$, which gives $\alpha_{1}^{2}=\frac{1}{3}, \alpha_{2}^{2}=\frac{1}{6}$, $\alpha_{3}^{2}=\frac{1}{6}$ and $\alpha_{4}^{2}=\frac{1}{3}$ respectively.

There are $n_{1}=2$ and $n_{2}=1$ for type 3 . Thus, $\alpha_{1}^{3}=\frac{2}{3}$ and $\alpha_{2}^{3}=\frac{1}{3}$.

Similarly for component type 4 , the data of $n_{1}=3, n_{2}=3, n_{3}=1$ and $n_{4}=1$ lead to $\alpha_{1}^{4}=\frac{3}{8}, \alpha_{2}^{4}=\frac{3}{8}, \alpha_{3}^{4}=\frac{1}{8}$ and $\alpha_{4}^{4}=\frac{1}{8}$.

There has an assumption that a common cause failure event occur will affect at least one component of each component type for the standard $\alpha$-factor model, therefore, it can be known for this system that $P(0,0,0,0)=P(0, b, c, d)=P(a, 0, c, d)=$ $P(a, b, 0, d)=P(a, b, c, 0)=0$, for $a=1,2,3, b=1,2,3,4, c=1,2$ and $d=1,2,3,4$. 
There is another assumption that the failure components of different types are independent, which implies that $P\left(f_{1}, f_{2}, f_{3}, f_{4}\right)=P\left(f_{1}\right) P\left(f_{2}\right) P\left(f_{3}\right) P\left(f_{4}\right)$. According to the conception, $P\left(f_{1}, f_{2}, f_{3}, f_{4}\right)$ is calculated by the available past data on the system, and is combined with the $\alpha$-factor model for common cause failures.

Recall that $\alpha_{1}^{1}$ represents the probability that exactly one component of the three components of type 1 failure. Since it has been assumed that one component of type 1 fails, it follows that $P\left(f_{1}\right)=\alpha_{1}^{1}$. So the alpha parameters provide all the information that required to specify the distribution. For instance, $P(1,1,1,1)=$ $\alpha_{1}^{1} \alpha_{1}^{2} \alpha_{1}^{3} \alpha_{1}^{4}=\frac{1}{4} * \frac{1}{3} * \frac{2}{3} * \frac{3}{8}=\frac{1}{48}$.

Then, it is necessary to calculate the survival signature $\Phi$, which is used to combine with the $P\left(f_{1}, f_{2}, f_{3}, f_{4}\right)$ to assess the survival probability of the system. For $P(1,1,1,1)$, its corresponding survival signature is $\Phi(2,3,2,3)$, which means that probability of the system works given that exact 2 components of type one, 3 components of type two, 2 components of type three and 3 components of type four are working. There are altogether 48 possible state vectors, of which 41 combinations allow the system to function. Therefore, $\Phi(2,3,2,3)=\frac{41}{48}$.

All the values of $P\left(f_{1}, f_{2}, f_{3}, f_{4}\right)$ and their corresponding survival signature $\Phi\left(m_{1}-f_{1}, m_{2}-f_{2}, m_{3}-f_{3}, m_{4}-f_{4}\right)$ can be calculated. Based on the values and Equation 6 , the probability that the complex system works after the next common cause failure event $P\left(T_{s}>t \mid C C F\right)$ is $\frac{50}{139}$.

\subsection{Case 2: general $\alpha$-factor model 1}

Let continue use the complex system in Figure 1, however, without assuming that the common cause event will affect all the common cause failure groups. Again, the component types form the common cause failure groups as before. Therefore, it can be any number combinations of the components from type 1 to type 4 . Note again that $P(0,0,0,0)=0$ as at least one component will be affected by the CCFs.

The past data on the system can be seen in Table 1, which has more data available to fit the requirements for implementing the general $\alpha$-factor model.

Table 1: Past data $n_{j_{1} j_{2} j_{3} j_{4}}$ on the system in Figure 1

\begin{tabular}{|l|l|l|l|l|l|}
\hline$n_{j_{1} j_{2} j_{3} j_{4}}$ & $n_{j_{1} j_{2} j_{3} j_{4}}$ & $n_{j_{1} j_{2} j_{3} j_{4}}$ & $n_{j_{1} j_{2} j_{3} j_{4}}$ & $n_{j_{1} j_{2} j_{3} j_{4}}$ & $n_{j_{1} j_{2} j_{3} j_{4}}$ \\
\hline$n_{0001}=1$ & $n_{0002}=1$ & $n_{0003}=1$ & $n_{0004}=1$ & $n_{0010}=1$ & $n_{0011}=1$ \\
$n_{0012}=1$ & $n_{0013}=1$ & $n_{0014}=1$ & $n_{0020}=1$ & $n_{0021}=1$ & $n_{0022}=1$ \\
$n_{0023}=1$ & $n_{0024}=1$ & $n_{0100}=1$ & $n_{0101}=1$ & $n_{0102}=3$ & $n_{0103}=1$ \\
$n_{0104}=1$ & $n_{0110}=1$ & $n_{0111}=1$ & $n_{0112}=1$ & $n_{0113}=1$ & $n_{0114}=1$ \\
$n_{0120}=1$ & $n_{0121}=1$ & $n_{0122}=1$ & $n_{0123}=1$ & $n_{0124}=2$ & $n_{0200}=1$ \\
$n_{0201}=1$ & $n_{0202}=1$ & $n_{0203}=1$ & $n_{0204}=1$ & $n_{0210}=1$ & $n_{0211}=2$ \\
$n_{0212}=1$ & $n_{0213}=1$ & $n_{0214}=1$ & $n_{0220}=1$ & $n_{0221}=1$ & $n_{0222}=1$ \\
$n_{0223}=1$ & $n_{0224}=1$ & $n_{0300}=1$ & $n_{0301}=1$ & $n_{0302}=1$ & $n_{0303}=1$ \\
$n_{0304}=1$ & $n_{0310}=2$ & $n_{0311}=2$ & $n_{0312}=1$ & $n_{0313}=1$ & $n_{0314}=1$ \\
\hline
\end{tabular}


October 22, 2018 13:5 WSPC/INSTRUCTION FILE ws-ijrqse

Table 1 - Continued from previous page

\begin{tabular}{|c|c|c|c|c|c|}
\hline$n_{j_{1} j_{2} j_{3} j_{4}}$ & $n_{j_{1} j_{2} j_{3} j_{4}}$ & $n_{j_{1} j_{2} j_{3} j_{4}}$ & $n_{j_{1} j_{2} j_{3} j_{4}}$ & $n_{j_{1} j_{2} j_{3} j_{4}}$ & $n_{j_{1} j_{2} j_{3} j_{4}}$ \\
\hline$n_{0320}=1$ & $n_{0321}=1$ & $n_{0322}=1$ & $n_{0323}=1$ & $n_{0324}=1$ & $n_{0400}=1$ \\
\hline$n_{0401}=1$ & $n_{0402}=1$ & $n_{0403}=1$ & $n_{0404}=1$ & $n_{0410}=1$ & $n_{0411}=2$ \\
\hline$n_{0412}=1$ & $n_{0413}=1$ & $n_{0414}=1$ & $n_{0420}=1$ & $n_{0421}=1$ & $n_{0422}=1$ \\
\hline$n_{0423}=1$ & $n_{0424}=1$ & $n_{1000}=1$ & $n_{1001}=1$ & $n_{1002}=1$ & $n_{1003}=1$ \\
\hline$n_{1004}=1$ & $n_{1010}=1$ & $n_{1011}=1$ & $n_{1012}=2$ & $n_{1013}=1$ & $n_{1014}=1$ \\
\hline$n_{1020}=1$ & $n_{1021}=1$ & $n_{1022}=1$ & $n_{1023}=1$ & $n_{1024}=1$ & $n_{1100}=1$ \\
\hline$n_{1101}=1$ & $n_{1102}=1$ & $n_{1103}=2$ & $n_{1104}=1$ & $n_{1110}=2$ & $n_{1111}=1$ \\
\hline$n_{1112}=1$ & $n_{1113}=1$ & $n_{1114}=1$ & $n_{1120}=1$ & $n_{1121}=1$ & $n_{1122}=1$ \\
\hline$n_{1123}=1$ & $n_{1124}=1$ & $n_{1200}=1$ & $n_{1201}=2$ & $n_{1202}=1$ & $n_{1203}=1$ \\
\hline$n_{1204}=1$ & $n_{1210}=1$ & $n_{1211}=1$ & $n_{1212}=1$ & $n_{1213}=1$ & $n_{1214}=1$ \\
\hline$n_{1220}=1$ & $n_{1221}=3$ & $n_{1222}=2$ & $n_{1223}=1$ & $n_{1224}=1$ & $n_{1300}=1$ \\
\hline$n_{1301}=1$ & $n_{1302}=1$ & $n_{1303}=1$ & $n_{1304}=1$ & $n_{1310}=1$ & $n_{1311}=3$ \\
\hline$n_{1312}=1$ & $n_{1313}=1$ & $n_{1314}=1$ & $n_{1320}=1$ & $n_{1321}=1$ & $n_{1322}=1$ \\
\hline$n_{1323}=1$ & $n_{1324}=1$ & $n_{1400}=1$ & $n_{1401}=1$ & $n_{1402}=1$ & $n_{1403}=1$ \\
\hline$n_{1404}=2$ & $n_{1410}=4$ & $n_{1411}=1$ & $n_{1412}=1$ & $n_{1413}=1$ & $n_{1414}=1$ \\
\hline$n_{1420}=1$ & $n_{1421}=1$ & $n_{1422}=1$ & $n_{1423}=1$ & $n_{1424}=1$ & $n_{2000}=1$ \\
\hline$n_{2001}=1$ & $n_{2002}=1$ & $n_{2003}=1$ & $n_{2004}=1$ & $n_{2010}=3$ & $n_{2011}=1$ \\
\hline$n_{2012}=1$ & $n_{2013}=1$ & $n_{2014}=1$ & $n_{2020}=1$ & $n_{2021}=1$ & $n_{2022}=1$ \\
\hline$n_{2023}=2$ & $n_{2024}=1$ & $n_{2100}=1$ & $n_{2101}=1$ & $n_{2102}=1$ & $n_{2103}=2$ \\
\hline$n_{2104}=1$ & $n_{2110}=1$ & $n_{2111}=1$ & $n_{2112}=1$ & $n_{2113}=1$ & $n_{2114}=1$ \\
\hline$n_{2120}=1$ & $n_{2121}=1$ & $n_{2122}=1$ & $n_{2123}=1$ & $n_{2124}=1$ & $n_{2200}=1$ \\
\hline$n_{2201}=2$ & $n_{2202}=1$ & $n_{2203}=1$ & $n_{2204}=1$ & $n_{2210}=1$ & $n_{2211}=1$ \\
\hline$n_{2212}=1$ & $n_{2213}=1$ & $n_{2214}=1$ & $n_{2220}=1$ & $n_{2221}=2$ & $n_{2222}=1$ \\
\hline$n_{2223}=1$ & $n_{2224}=3$ & $n_{2300}=1$ & $n_{2301}=1$ & $n_{2302}=1$ & $n_{2303}=1$ \\
\hline$n_{2304}=1$ & $n_{2310}=1$ & $n_{2311}=1$ & $n_{2312}=1$ & $n_{2313}=1$ & $n_{2314}=1$ \\
\hline$n_{2320}=1$ & $n_{2321}=1$ & $n_{2322}=1$ & $n_{2323}=1$ & $n_{2324}=1$ & $n_{2400}=1$ \\
\hline$n_{2401}=1$ & $n_{2402}=2$ & $n_{2403}=1$ & $n_{2404}=1$ & $n_{2410}=1$ & $n_{2411}=1$ \\
\hline$n_{2412}=1$ & $n_{2413}=1$ & $n_{2414}=1$ & $n_{2420}=1$ & $n_{2421}=1$ & $n_{2422}=1$ \\
\hline$n_{2423}=1$ & $n_{2424}=1$ & $n_{3000}=2$ & $n_{3001}=1$ & $n_{3002}=2$ & $n_{3003}=1$ \\
\hline$n_{3004}=1$ & $n_{3010}=1$ & $n_{3011}=1$ & $n_{3012}=1$ & $n_{3013}=1$ & $n_{3014}=1$ \\
\hline$n_{3020}=1$ & $n_{3021}=1$ & $n_{3022}=1$ & $n_{3023}=3$ & $n_{3024}=1$ & $n_{3100}=1$ \\
\hline$n_{3101}=1$ & $n_{3102}=1$ & $n_{3103}=1$ & $n_{3104}=1$ & $n_{3110}=1$ & $n_{3111}=1$ \\
\hline$n_{3112}=2$ & $n_{3113}=1$ & $n_{3114}=1$ & $n_{3120}=1$ & $n_{3121}=1$ & $n_{3122}=1$ \\
\hline$n_{3123}=1$ & $n_{3124}=1$ & $n_{3200}=1$ & $n_{3201}=1$ & $n_{3202}=1$ & $n_{3203}=1$ \\
\hline$n_{3204}=1$ & $n_{3210}=1$ & $n_{3211}=3$ & $n_{3212}=1$ & $n_{3213}=1$ & $n_{3214}=1$ \\
\hline$n_{3220}=1$ & $n_{3221}=1$ & $n_{3222}=2$ & $n_{3223}=1$ & $n_{3224}=1$ & $n_{3300}=1$ \\
\hline$n_{3301}=1$ & $n_{3302}=1$ & $n_{3303}=1$ & $n_{3304}=2$ & $n_{3310}=1$ & $n_{3311}=1$ \\
\hline$n_{3312}=1$ & $n_{3313}=1$ & $n_{3314}=3$ & $n_{3320}=1$ & $n_{3321}=1$ & $n_{3322}=1$ \\
\hline$n_{3323}=2$ & $n_{3324}=2$ & $n_{3400}=1$ & $n_{3401}=1$ & $n_{3402}=1$ & $n_{3403}=2$ \\
\hline$n_{3404}=1$ & $n_{3410}=3$ & $n_{3411}=1$ & $n_{3412}=1$ & $n_{3413}=1$ & $n_{3414}=1$ \\
\hline
\end{tabular}

Continued on next page 
Table 1 - Continued from previous page

\begin{tabular}{|c|c|c|c|c|c|}
\hline$n_{j_{1} j_{2} j_{3} j_{4}}$ & $n_{j_{1} j_{2} j_{3} j_{4}}$ & $n_{j_{1} j_{2} j_{3} j_{4}}$ & $n_{j_{1} j_{2} j_{3} j_{4}}$ & $n_{j_{1} j_{2} j_{3} j_{4}}$ & $n_{j_{1} j_{2} j_{3} j_{4}}$ \\
\hline$n_{3420}=1$ & $n_{3421}=1$ & $n_{3422}=1$ & $n_{3423}=1$ & $n_{3424}=1$ & \\
\hline
\end{tabular}

$\sum_{j_{1}=0}^{3} \sum_{j_{2}=0}^{4} \sum_{j_{3}=0}^{2} \sum_{j_{4}=0}^{4} n_{j_{1} j_{2} j_{3} j_{4}}=345$ is got according to the above table, and the $\alpha$-factor parameters can be easily obtained by Equation 7. For instance, $\alpha_{2314}=\frac{1}{345}$. Since there is no assumption that independence among the components, the joint probability distribution $P\left(f_{1}, f_{2}, f_{3}, f_{4}\right)$ is simply given by the $\alpha$-factor parameters, which means $P(2,3,1,4)=\alpha_{2314}=\frac{1}{345}$.

The survival signature of the complex system in Figure 1 is totally the same as calculated before as it only depends on the structure of the system. Combining the joint probability distribution values and their corresponding survival signature, the survival probability of the system after the CCfs $P\left(T_{s}>t \mid C C F\right)$, which can be calculated by Equation 6, is $\frac{461}{934}$. This value is still low, however, it has an improvement compared with the initial failure event in Case 1.

\subsection{Case 3: general $\alpha$-factor model 2}

It can be seen from Case 2 that this system is highly susceptible to common cause failures, as all combinations of the failed components have occurred in the past data. There is a more robust system to be analysed in this Case, with past data can be seen in Table 2 .

Table 2: Past data $n_{j_{1} j_{2} j_{3} j_{4}}$ on the system in Figure 1

\begin{tabular}{|c|c|c|c|c|c|}
\hline$n_{j_{1} j_{2} j_{3} j_{4}}$ & $n_{j_{1} j_{2} j_{3} j_{4}}$ & $n_{j_{1} j_{2} j_{3} j_{4}}$ & $n_{j_{1} j_{2} j_{3} j_{4}}$ & $n_{j_{1} j_{2} j_{3} j_{4}}$ & $n_{j_{1} j_{2} j_{3} j_{4}}$ \\
\hline$n_{0001}=16$ & $n_{0002}=11$ & $n_{0003}=13$ & $n_{0004}=6$ & $n_{0010}=13$ & $n_{0011}=3$ \\
$n_{0012}=2$ & $n_{0013}=3$ & $n_{0014}=3$ & $n_{0020}=13$ & $n_{0021}=5$ & $n_{0022}=2$ \\
$n_{0023}=4$ & $n_{0100}=13$ & $n_{0101}=3$ & $n_{0102}=3$ & $n_{0103}=2$ & $n_{0104}=2$ \\
$n_{0110}=2$ & $n_{0111}=1$ & $n_{0120}=3$ & $n_{0200}=13$ & $n_{0201}=4$ & $n_{0202}=2$ \\
$n_{0203}=1$ & $n_{0204}=13$ & $n_{0210}=1$ & $n_{0220}=3$ & $n_{0300}=14$ & $n_{0301}=5$ \\
$n_{0302}=2$ & $n_{0303}=13$ & $n_{0304}=1$ & $n_{0310}=3$ & $n_{0320}=2$ & $n_{0400}=7$ \\
$n_{0401}=3$ & $n_{0402}=3$ & $n_{0403}=2$ & $n_{0404}=1$ & $n_{0410}=1$ & $n_{0420}=3$ \\
$n_{1000}=17$ & $n_{1001}=6$ & $n_{1002}=3$ & $n_{1003}=4$ & $n_{1004}=4$ & $n_{1010}=5$ \\
$n_{1011}=1$ & $n_{1020}=3$ & $n_{1100}=6$ & $n_{1101}=2$ & $n_{1102}=1$ & $n_{1110}=2$ \\
$n_{1111}=1$ & $n_{1200}=3$ & $n_{1201}=1$ & $n_{1300}=6$ & $n_{1301}=1$ & $n_{1400}=4$ \\
$n_{1401}=1$ & $n_{1402}=1$ & $n_{2000}=13$ & $n_{2001}=5$ & $n_{2002}=3$ & $n_{2003}=2$ \\
$n_{2004}=2$ & $n_{2010}=4$ & $n_{2011}=1$ & $n_{2020}=4$ & $n_{2100}=6$ & $n_{2101}=1$ \\
$n_{2110}=1$ & $n_{2200}=3$ & $n_{2300}=3$ & $n_{2301}=1$ & $n_{3000}=9$ & $n_{3001}=5$ \\
$n_{3002}=3$ & $n_{3010}=4$ & $n_{3020}=2$ & $n_{3100}=3$ & $n_{3300}=1$ & $n_{3301}=1$ \\
\hline
\end{tabular}


As can be seen from the above table that there is one past event $\left(n_{1111}\right)$ that all component types are affected, and few past data where three types have been influenced $\quad\left(n_{0111}, n_{1011}, n_{1101}, n_{1102}, n_{1110}, n_{1201}, n_{1401}, n_{1402}, n_{2011}, n_{2101}, n_{2110}\right.$, $n_{2301}, n_{3301}$ in this case). In other words, this system has the feature that components belong to one type are more reliable when CCFs affect the other types. Therefore, it is unlikely to have large numbers of components of more than two types fail simultaneously.

Similarly, $\sum_{j_{1}=0}^{3} \sum_{j_{2}=0}^{4} \sum_{j_{3}=0}^{2} \sum_{j_{4}=0}^{4} n_{j_{1} j_{2} j_{3} j_{4}}=345$ and the joint probability distribution $P\left(f_{1}, f_{2}, f_{3}, f_{4}\right)$ can be got directly from $\alpha$-factor parameters, with $P\left(f_{1}, f_{2}, f_{3}, f_{4}\right)=\alpha_{n_{1} n_{2} n_{3} n_{4}}$. The survival signature of the system remains the same as before since its structure does not change at all.

Based on Equation 6, it can be known that $P\left(T_{s}>t \mid C C F\right)=\frac{439}{473}$, which means the system survival probability after a common cause failure is around $92.81 \%$. This is highly improved compared with the former two cases.

\subsection{Case 4: imprecision within system}

In this subsection, let consider uncertainty in the system reliability analysis after common cause failures. In Case 1, if the system has not suffered CCF in the past, there might not be enough data to calculate the $\alpha$-factor parameters, although the standard $\alpha$-factor model can still be implemented by using experts' judgements.

Given that a total of 20 common cause component failures has occurred across the complex system in Figure 1, let two groups of experts estimate how the data would be spread. Suppose group one gives that $\alpha_{1}^{1}=\frac{2}{5}, \alpha_{2}^{1}=\frac{1}{5}$ and $\alpha_{3}^{1}=\frac{2}{5}$ for components type 1 , while for components type $2, \alpha_{1}^{2}=0, \alpha_{2}^{2}=\frac{2}{5}, \alpha_{3}^{2}=\frac{2}{5}$ and $\alpha_{4}^{2}=$ $\frac{1}{5} \cdot \alpha_{1}^{3}=\frac{2}{3}$ and $\alpha_{2}^{3}=\frac{1}{3}$ for components type 3 , and for components type $4, \alpha_{1}^{4}=\frac{3}{7}$, $\alpha_{2}^{4}=\frac{1}{7}, \alpha_{3}^{4}=\frac{2}{7}$ and $\alpha_{4}^{4}=\frac{1}{7}$. At this time, even the survival signature of $\Phi(2,3,2,3)$ remains the same as $\frac{41}{48}$, which is a big value within the survival signature. However, its corresponding $P(1,1,1,1)$ decreases from $\frac{1}{48}$ to 0 . Let summarise the products of $P\left(f_{1}, f_{2}, f_{3}, f_{4}\right)$ and their corresponding $\Phi\left(m_{1}-f_{1}, m_{2}-f_{2}, m_{3}-f_{3}, m_{4}-f_{4}\right)$, then the survival probability $P\left(T_{s}>t \mid C C F\right)$ of the complex system after the next CCFs is $\frac{6}{23}$.

For group two, the estimated data are $\alpha_{1}^{1}=\frac{1}{2}, \alpha_{2}^{1}=\frac{1}{3}, \alpha_{3}^{1}=\frac{1}{6}, \alpha_{1}^{2}=\frac{1}{4}, \alpha_{2}^{2}=\frac{1}{4}$, $\alpha_{3}^{2}=\frac{1}{4}, \alpha_{4}^{2}=\frac{1}{4}, \alpha_{1}^{3}=\frac{1}{2}, \alpha_{2}^{3}=\frac{1}{2}, \alpha_{1}^{4}=\frac{3}{8}, \alpha_{2}^{4}=\frac{3}{8}, \alpha_{3}^{4}=\frac{1}{4}$ and $\alpha_{4}^{4}=0$. For this circumstance, $P(1,1,1,1)$ increases to $\alpha_{1}^{1} \alpha_{1}^{2} \alpha_{1}^{3} \alpha_{1}^{4}=\frac{1}{2} * \frac{1}{4} * \frac{1}{2} * \frac{3}{8}=\frac{3}{128}$. Therefore, $P\left(T_{s}>t \mid C C F\right)=\frac{179}{456}$ according to Equation 6 .

So due to the epistemic uncertainty in this example, the probability bounds of the system works after the next common cause failure event is $\underline{\underline{P}}\left(T_{s}>t \mid C C F\right)=$ $\left[\frac{6}{23}, \frac{3}{128}\right]$.

\subsection{Results discussion}

Common cause failures are the simultaneous failure of multiple similar components 
due to the same root cause, these kind of failures have been shown to decrease the reliabiliyt and performance of complex systems. ${ }^{33}$ A common cause failure event may affect an entire system or only a fraction of its components. Consequently, the number of components involved in a common cause failure event ranges from 1 to the total number of components within the same type.

In Case 1, due to the assumptions of the standard $\alpha$-factor model, the survival probability of the system is unsatisfactorily low. In order to release these assumptions, it is necessary to generalise the $\alpha$-factor model. Both Case 2 and Case 3 improve from $\frac{50}{139}$ to $\frac{461}{934}$ and $\frac{439}{473}$, respectively. The structure of the system remains the same for case 2 and case there, and the total number of the past data is identical to Case 2, but why the vulnerability of the system to CCFs declines so drastically? It can be investigated that the higher values of $P\left(f_{1}, f_{2}, f_{3}, f_{4}\right), \frac{17}{345}, \frac{16}{345}, \frac{14}{345}, \frac{13}{345}$ and $\frac{11}{345}$, correspond to survival signature $\Phi\left(l_{1}, l_{2}, l_{3}, l_{4}\right)=1$. Therefore, these kinds of CCFs that are triggered will not be likely to cause system fails. While in Case 2 , there are more instances in the past data where larger numbers of components from more than two types had failed, making it less likely that the system could continue to work. Case 4 shows that epistemic uncertainty will lead the uncertainty within the system in this case. In order to reduce the imprecision, engineers need to put efforts to get the precise $\alpha$-factor parameters.

\section{Conclusions}

Common cause failure events have highly adverse impact on the reliability and availability of systems in the real world, which makes it important to analyse systems reliability after CCFs. This paper put forwards the survival signature based reliability analysis on complex systems with common cause failures.

The survival signature is a summary of system structure function, which makes it is efficient to analyse complex systems. Based on the basic $\alpha$-factor model, the paper presents the standard and general $\alpha$-factor models for analysing complex system reliability with common cause failures. The effects of epistemic uncertainty are taken into account as well. What is more, the proposed methods are combined with the survival signature in order to perform complex system reliability analysis in the presence of common cause failure events. The feasibility and effectiveness of the proposed measures are demonstrated by the numerical examples.

The standard $\alpha$-factor model has the assumptions that (1) the common cause failure event will affect all the common cause groups of components; and (2) the failure components are independent. All of which may lead to an unsatisfactory survival probability of system after a common cause failure event. In practice, the engineer will have to consider repairing or replacing the failed components if this kind of circumstance happens. Therefore, the CCFs model combines with the survival signature can be used to decide whether to repair or replace the failed components immediately, or after the next common cause failure event. In other words, the administrator or designer has to consider the cost of repairing or replace 
the components as soon as possible, or taking the risk of allowing next common cause failure vent to occur before performing the failed components repair or replacement. Overall, it is an example of how decision theory is incorporated in the practical engineering world.

In order to release the assumptions of the former model, the general $\alpha$-factor model is introduced. By investigating the two cases which are given for this model, it can be seen that design for reliability is useful in the engineering world. The goal of the reliability policy is to achieve high initial reliability by focusing on reliability fundamentals during design. So design for reliability provides engineers and managers with a range of tools and techniques for incorporating reliability into the design process for complex systems. ${ }^{34}$ For complex system, it can examine the survival signature first, in order to find out which component combinations of failures will not allow the system to function at all. Therefore, the engineers can ensure these components are equipped to be more reliable under certain circumstances, which makes the system more reliable.

A possible challenge of the work presented in this paper is that there may not be enough past data on the realistic system, which is essential to calculate the precise values of the $\alpha$-factor parameters. This is either if the system does not usually experience the common cause failures, or if the system has not been run in the past. However, these $\alpha$-factor models can still be implemented by using experts' judgements on the system to ascertain the $n_{j_{1}, j_{2}, \ldots, j_{K}}$ values.

\section{Acknowledgement}

Geng Feng would like to thank Professor Frank P.A. Coolen for discussion and the initial work which has done by Ms Roshni Prasad. The author wishes to thank the anonymous reviewers for their valuable comments which improved the paper.

\section{Declaration of Conflicting Interests}

The authors declare no potential conflicts of interest with respect to the research, authorship, and/or publication of this article.

\section{References}

1. G. Vining, M. Kulahci and S. Pedersen, Recent advances and future directions for quality engineering, Quality and Reliability Engineering International 32(3) (2016) 863-875.

2. M. Modarres, Risk analysis in engineering: techniques, tools, and trends (CRC press, 2006).

3. D. M. Rasmuson and D. L. Kelly, Common-cause failure analysis in event assessment, Proceedings of the Institution of Mechanical Engineers, Part O: Journal of Risk and Reliability 222(4) (2008) 521-532.

4. K. Fleming, Reliability model for common mode failures in redundant safety systems, in Modeling and simulation. Volume 6, Part 1, 1975 
5. K. N. Fleming, A. Mosleh and R. K. Deremer, A systematic procedure for the incorporation of common cause events into risk and reliability models, Nuclear Engineering and Design 93(2-3) (1986) 245-273.

6. A. Mosleh, K. Fleming, G. Parry, H. Paula, D. Worledge and D. M. Rasmuson, Procedures for treating common cause failures in safety and reliability studies: Volume 1, procedural framework and examples: Final report, tech. rep., Pickard, Lowe and Garrick, Inc., Newport Beach, CA (USA) (1988).

7. A. Mosleh, Common cause failures: an analysis methodology and examples, Reliability Engineering \& System Safety 34(3) (1991) 249-292.

8. C. L. Atwood, The binomial failure rate common cause model, Technometrics 28(2) (1986) 139-148.

9. W. Li and M. J. Zuo, Reliability evaluation of multi-state weighted k-out-of-n systems, Reliability engineering \& system safety 93(1) (2008) 160-167.

10. S. Eryilmaz and G. Y. Tutuncu, Reliability evaluation of linear consecutive-weightedk-out-of-n: F system, Asia-Pacific Journal of Operational Research 26(06) (2009) $805-816$.

11. D. Kelly and C. Atwood, Finding a minimally informative dirichlet prior distribution using least squares, Reliability Engineering \&3 System Safety 96(3) (2011) 398-402.

12. C. Wang, L. Xing and G. Levitin, Explicit and implicit methods for probabilistic common-cause failure analysis, Reliability Engineering \& System Safety 131 (2014) $175-184$.

13. H. George-Williams, G. Feng, F. P. Coolen, M. Beer and E. Patelli, Extending the survival signature paradigm to complex systems with non-repairable dependent failures., Proceedings of the Institution of Mechanical Engineers, part O: journal of risk and reliability. (2019).

14. G. Levitin, Incorporating common-cause failures into nonrepairable multistate seriesparallel system analysis, IEEE Transactions on Reliability 50(4) (2001) 380-388.

15. M. C. Troffaes, G. Walter and D. Kelly, A robust bayesian approach to modeling epistemic uncertainty in common-cause failure models, Reliability Engineering 85 System Safety 125 (2014) 13-21.

16. F. P. Coolen and T. Coolen-Maturi, Predictive inference for system reliability after common-cause component failures, Reliability Engineering \& System Safety 135 (2015) 27-33.

17. F. P. Coolen and T. Coolen-Maturi, Generalizing the signature to systems with multiple types of components, in Complex Systems and Dependability, (Springer, 2012) pp. $115-130$.

18. F. J. Samaniego, System signatures and their applications in engineering reliability (Springer Science \& Business Media, 2007).

19. F. P. Coolen and T. Coolen-Maturi, Modelling uncertain aspects of system dependability with survival signatures, in Dependability Problems of Complex Information Systems, (Springer, 2015) pp. 19-34.

20. F. P. Coolen and T. Coolen-Maturi, On the structure function and survival signature for system reliability, in Safety and Reliability, Taylor \& Francis2016, pp. 1-11.

21. L. J. Aslett, Reliabilitytheory: Tools for structural reliability analysis. $\mathrm{r}$ package (2012).

22. L. J. Aslett, F. P. Coolen and S. P. Wilson, Bayesian inference for reliability of systems and networks using the survival signature, Risk Analysis 35(3) (2015) 1640-1651.

23. F. P. Coolen, T. Coolen-Maturi and A. H. Al-Nefaiee, Nonparametric predictive inference for system reliability using the survival signature, Proceedings of the Institution of Mechanical Engineers, Part O: Journal of Risk and Reliability 228(5) (2014) 437- 
448.

24. G. Feng, E. Patelli, M. Beer and F. P. Coolen, Imprecise system reliability and component importance based on survival signature, Reliability Engineering \&5 System Safety 150 (2016) 116-125.

25. G. Feng, Sensitivity analysis for systems under epistemic uncertainty with probability bounds analysis, International Journal of Computer Applications 179(31) (2018) 1-6, doi:10.5120/ijca2018915892.

26. G. Walter, L. J. Aslett and F. Coolen, Bayesian nonparametric system reliability using sets of priors, International Journal of Approximate Reasoning 30 (2017) 67-88.

27. E. Patelli, G. Feng, F. P. Coolen and T. Coolen-Maturi, Simulation methods for system reliability using the survival signature, Reliability Engineering 83 System Safety 167 (2017) 327-337.

28. S. Reed, An efficient algorithm for exact computation of system and survival signatures using binary decision diagrams, Reliability Engineering \& System Safety 165 (2017) 257-267.

29. S. Eryilmaz, F. P. Coolen and T. Coolen-Maturi, Marginal and joint reliability importance based on survival signature, Reliability Engineering \&3 System Safety 172 (2018) 118-128.

30. S. Eryilmaz, F. P. Coolen and T. Coolen-Maturi, Mean residual life of coherent systems consisting of multiple types of dependent components, Naval Research Logistics (NRL) 65(1) (2018) 86-97.

31. A. Mosleh, D. M. Rasmuson and F. Marshall, Guidelines on modeling common-cause failures in probabilistic risk assessment (Safety Programs Division, Office for Analysis and Evaluation of Operational Data, US Nuclear Regulatory Commission, 1998).

32. K. Yury, G. Lera, K. Elena, V. Irina and D. Sergey, Consideration of uncertainties and risks in the building process of multifunctional harbor transshipment complex, International Journal of Reliability, Quality and Safety Engineering 23(06) (2016) p. 1640011.

33. B. Dhillon and O. Anude, Common-cause failures in engineering systems: A review, International Journal of Reliability, Quality and Safety Engineering 1(01) (1994) 103129.

34. D. G. Raheja and L. J. Gullo, Design for reliability (John Wiley \& Sons, 2012).

\begin{abstract}
About the Author
Dr. Geng Feng is a Lecturer in the School of Engineering, University of Central Lancashire, United Kingdom. He has been working as a researcher in University of Bristol and Thales UK Ltd after he obtained his PhD degree from University of Liverpool in 2017. His main research interests include RAMS (Reliability, Availability, Maintainability and Safety) of complex systems, imprecision theory, sensitivity analysis and component importance measures.
\end{abstract}

